\title{
Effects of fatigue on center-of-mass acceleration during a prolonged overground run
}

\author{
Scott Evans ${ }^{1 *}$, Sara Winter ${ }^{2}$ \\ ${ }^{1}$ Physiotherapist, James Cook University, Australia \\ ${ }^{2}$ Musculoskeletal Physiotherapy, James Cook University, Australia
}

\begin{abstract}
Objective: The aim of this study was to assess for fatigue related changes in center-of-mass (COM) acceleration during a prolonged overground run in a group of healthy male runners using a tri-axial accelerometer attached to a runner's low back.

Methods: Thirty healthy male runners' performed an eight kilometer (20 laps) overground time trial paced run on a 400 meter athletics track. Kinematic measures assessed regularly throughout the run included signal energy and acceleration impulse in the anteroposterior (AP), mediolateral (ML) and vertical (VT) directions, contact time (CT) and step frequency. A repeated measures analysis of variance comparing each data collection point to the initial was used to identify fatigue related changes.

Results: Compared to initial measurement, CT significantly increased during laps six to 18 in addition to significant increases in ML signal energy and decreases in AP impulse identified from lap four to 20. Conclusion: A tri-axial accelerometer was able to identify changes in ML and AP COM acceleration measures plus a runner's $C T$ indicating fatigue was impacting a runner throughout a prolonged run. Limited changes in VT acceleration measures were identified suggesting biomechanical fatigue related running changes predominately occur in the horizontal plane. Future research investigating the effects of fatigue on running should use frequent and regular data collection to identify fatigue related running changes.
\end{abstract}

Keywords: Accelerometry, Kinematics, Running, Biomechanics

Accepted on January 29, 2018

\section{Introduction}

Distance running has become a popular sport and recreational activity in modern day society. Despite its popularity, distance running results in a high prevalence of injuries, with up to $70 \%$ of runners sustaining a running related injury during a one-year period [1]. Running in a fatigued state has been reported to alter running kinematics [2-4]. Fatigue related changes in running kinematics have been suggested to increase the risk of injury [5] and negatively impact a runner's performance [2].

Assessment of fatigue changes during running are commonly performed on a treadmill [6,7]. Running on a treadmill may not be a true representation of overground running, especially during prolonged running with fatigue related changes occurring [8]. Tri-axial accelerometers are a small, lightweight device that allow for continuous monitoring of running kinematics in-field conditions, making them a highly practical tool for analysing running movement in overground conditions $[3,8,9]$. A single tri-axial accelerometer placed on a runner's low back has been shown to be a reliable [10] and valid [11] method of measuring center-of-mass (COM) accelerations. This method has the capacity to detect changes in kinematics with fatigue in overground running [3,9]. However limitations of the studies investigating overground kinematics have been limited by inconsistent frequency of data collection and analysis.
Girard et al. [2] performed frequent and regular interval data collection and analysis (every 200 meters (m) of a five kilometer $(\mathrm{km})$ run) and reported fatigue changes in movement from as early on as $200 \mathrm{~m}$. Other studies have performed less frequent data analysis $[3,4,12]$ which indicates that some running protocols may not have identified all possible fatigue related running changes. This may impact the accuracy and usability of results identified. Accurately identifying where fatigue impacts a runner during a prolonged distance run may allow for the implementation of fatigue minizing running strategies to ultimately increase running performance/ decreased the risk of a running related injury.

A single tri-axial accelerometer, with the capability to continuously collect data and allow for frequent analysis, can be utilized to understand more about fatigue related changes throughout a distance run. Therefore, the aim of this study was to measure COM acceleration to identify changes in running kinematics during a prolonged overground run using a single tri-axial accelerometer in a group of healthy long distance runners. 


\section{Methods}

\section{Design and participants}

This was an observational repeated measures study. Thirty male recreational and competitive runners were recruited through contact with the local running club (Townsville Road Runners), social media, emails and word of mouth. To be included participants had to be running at least $30 \mathrm{~km}$ a week, running regularly for at least a year and be injury free at the time of data collection. Participants were excluded if they had any neurological deficits, known lower limb deformities, medical conditions that may affect their running or were sensitive to adhesive sprays or double sided tape. Participants gave written informed consent prior to the run and the study was approved by the James Cook University Ethics Committee (H6068).

\section{Procedures}

A single tri-axial accelerometer $(52 \mathrm{~mm} \times 30 \mathrm{~mm} \times 13 \mathrm{~mm}$, mass $23 \mathrm{~g}$; resolution 16-bit, full-scale range $16 \mathrm{~g}$, sampling at $250 \mathrm{~Hz}$; SABEL Labs, Brisbane, Australia) was firmly fixated to a participants low back at L5/S1 level $[9,11]$, using adhesive spray, double sided foam tape and an elastic waist band. Data was collected by the sensor and wirelessly transferred to a computer for further analysis. Calibration was performed for each accelerometer as per the instruction manual prior to data collection. The prolonged run was an eight $\mathrm{km}$ self-selected time trial pace run on an outdoor $400 \mathrm{~m}$ all-weather athletics track. Participants wore their own running clothing and performed their own warm-up prior to the run. The runner's time was recorded each time they passed the $70 \mathrm{~m}$ mark on the home straight of the track. This ensured that data was analyzed on the "straight" of the track, as running movement may be altered when running around the bend of the track.

\section{Data analysis}

Acceleration data was analyzed using MATLAB R2015a software. Sixteen consecutive steps from the $70 \mathrm{~m}$ point on the home straight of the running track was analyzed every second lap or $800 \mathrm{~m}$ (that is $730 \mathrm{~m}, 1530 \mathrm{~m}, 2330 \mathrm{~m}$.... $7930 \mathrm{~m}$ ) using MATLAB R2015a software. Kinematic variables analyzed from the COM acceleration data included signal energy and impulse in the anteroposterior (AP), mediolateral (ML) and vertical (VT) directions. In addition, contact time (CT) and step frequency (SF) were measured. Step frequency is the number of steps a runner takes each minute, and was calculated by manually plotting peak VT accelerations. Contact time was calculated by plotting heel strike and toe off in the acceleration data. Heel strike corresponded to the beginning of the downward spike of the AP acceleration data and toe off was represented by the VT acceleration data crossing zero from an upward curve [11] (Figure 1). Reliability was determined prior to data analysis for manually plotting peak VT acceleration, heel strike and toe off with standard error of measurement calculated to be 0 .

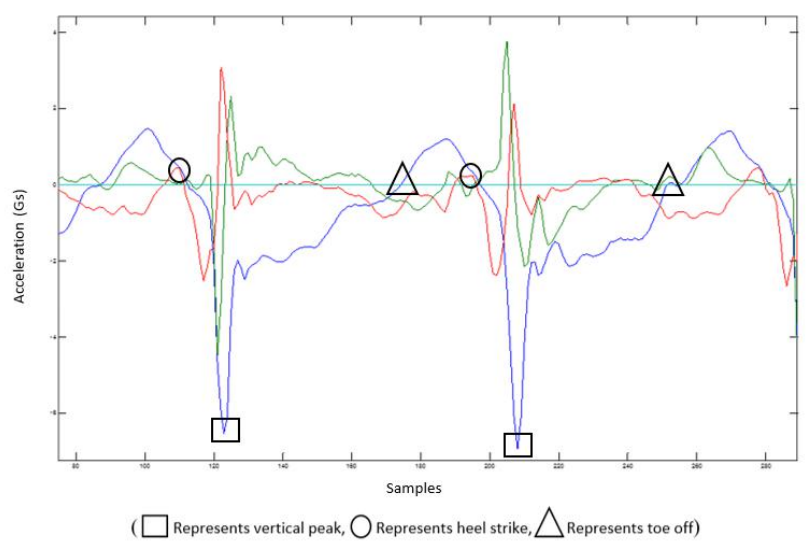

Figure 1. Heel strike, toe off and peak vertical accelerations in the acceleration data for two consecutive steps.

Acceleration impulse and signal energy was calculated for all acceleration directions. Signal energy is an indicator of metabolic energy expenditure related to acceleration [13], and was calculated from finding the integrals of the squared value of the accelerometer output [3].

$E=\int_{t=0}^{T} a^{2} d t$

Where a (acceleration $)=[a x, a y, a z]$ measured in gravity $(\mathrm{g})$ and $\mathbf{E}($ signal energy $)=[E x, E y, E z]$ measured in $\mathrm{g} 2 . \mathrm{s}$

Acceleration impulses were estimated from Newton's second law $($ Force $=$ Mass $\times$ Acceleration $)$. Linear impulses, the integral of force overtime, were calculated by the integral under the respective force time curves [3].

$I=\int_{t=0}^{T} F d t$

Where $\mathbf{F}($ force $)=[F x, F y, F z]$ measured in $\mathrm{N}$ (newtons) and $\mathbf{I}$ (impulse $)=[I x, I y, I z]$ measured in N.s

A low pass filter of $10 \mathrm{~Hz}$ was applied to smooth the acceleration data by eliminating small background noises. This filter has previously been used when validating tri-axial accelerometers to a motion analysis system [11].

\section{Statistical analysis}

Statistical analysis was performed using IBM SPPS statistics version 23. Variables were expressed as means \pm standard deviation (SD) and normality of distribution was tested using Kolmogrov-Smirnov test. Mauchly's test verified sphericity and when the assumption of sphericity was not met; the significance of F-ratios was adjusted according to the Greenhouse-Geisser procedure. A repeated measure Analysis of Variance (ANOVA) which incorporated a simple contrast analysis comparing each measurement to the initial measurement $(770 \mathrm{~m})$ was performed to determine fatigue 
Citation: Evans S, Winter S. Effects of fatigue on center-of-mass acceleration during a prolonged overground run. J Phys Ther Sports Med

related changes over time. The level of significance was set at $\alpha=0.05$.

\section{Results}

Participants characteristics (mean \pm SD) were height $(177.9 \mathrm{~cm}$ $\pm 7.78)$, weight $(77.13 \mathrm{~kg} \pm 8.86)$ and age $(37.5$ years \pm 9.4$)$. A significant decrease in VT impulse occurred at lap 20 only $(\mathrm{P}=0.02)$. Impulse in AP acceleration significantly decreased from laps 4 to 20 when compared the initial measurement $(\mathrm{P}<0.05)$. Greatest change in AP impulse was identified at lap 16 (1186.61 \pm 465.50 N.s $)(\mathrm{P}=0.002)$. No significant change was detected for ML impulse (Figure 2).

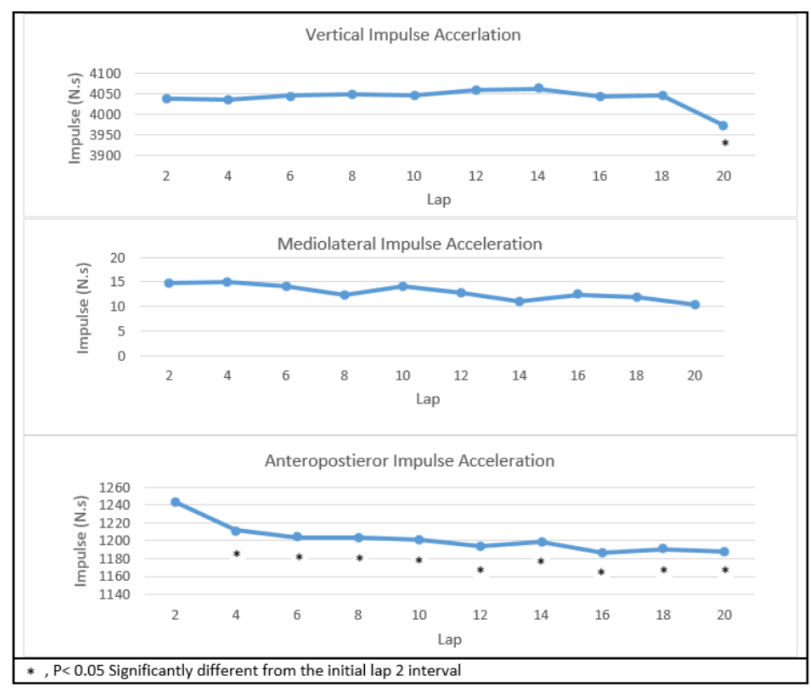

Figure 2. Acceleration Impulse for AP, ML and VT Acceleration for laps two to 20 of the eight $\mathrm{km}$ run.

Significant increases in ML signal energy were detected from laps four to $20(\mathrm{P}<0.05)$, with the greatest increase identified at lap $20(2.7157 \pm 1.086 \mathrm{~g} 2 . \mathrm{s})(\mathrm{P}=0.001)$. Significant decreases in AP signal energy were identified at lap four $(\mathrm{P}=0.041)$ and lap $20(\mathrm{P}=0.22)$. No significant changes were observed for VT signal energy (Figure 3 ).

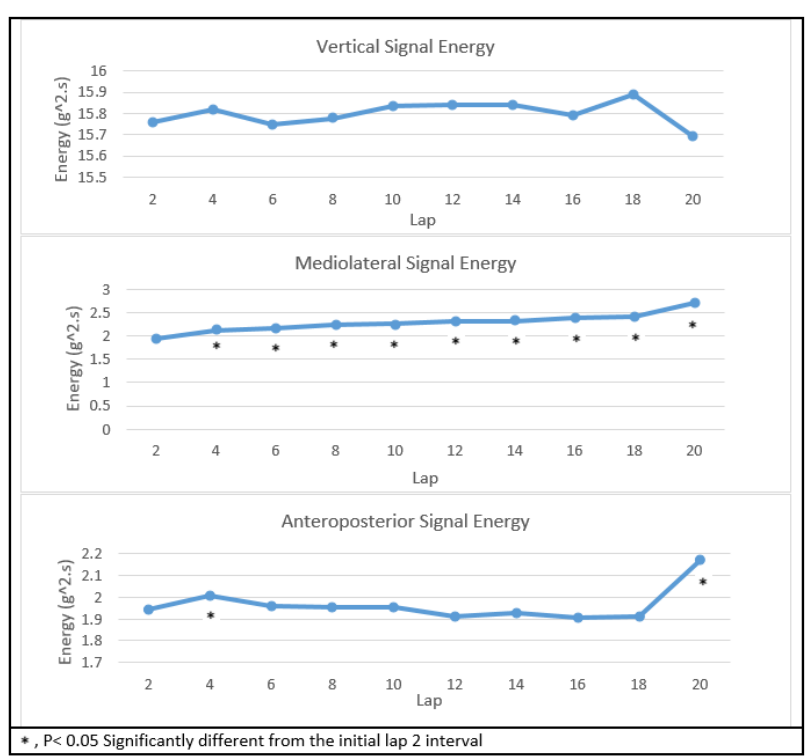

Figure 3. Signal Energy for AP, ML and VT Acceleration for laps two to 20 of the eight $\mathrm{km}$ run.

Significant increases in CT were identified for laps six to 18 $(\mathrm{P}<0.05)$ with greatest change observed in lap $12(0.228 \pm 0.26$ sec) $(\mathrm{P}=0.001)$. A significant increase in $\mathrm{SF}$ was observed in lap 20 only ( $\mathrm{P}=0.01)$ (Figure 4).

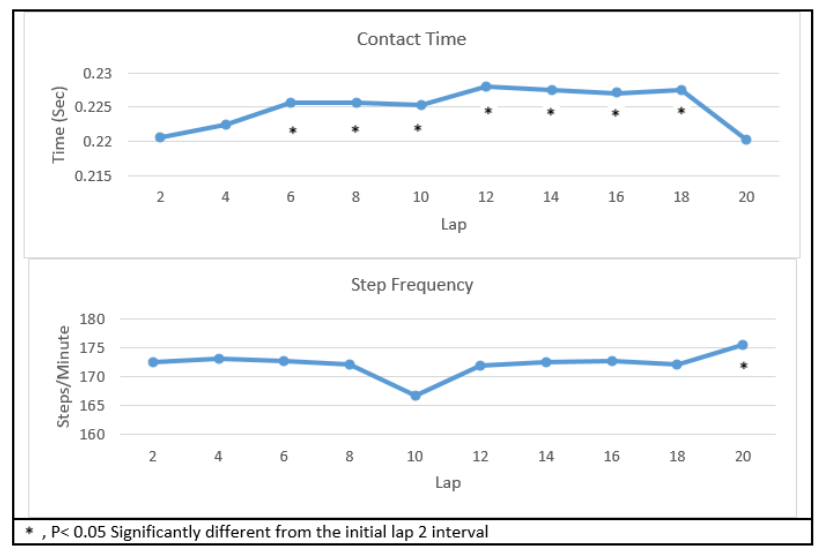

Figure 4. Contact time and Step Frequency for laps two to 20 of the eight $\mathrm{km}$ run.

\section{Discussion}

A single tri-axial accelerometer was able to identify changes in COM acceleration during a self-selected paced prolonged run. Significant changes occurred from as early as the fourth lap $(1530 \mathrm{~m})$ of the prolonged run and continued until the end of the run, indicating fatigue related changes in running kinematics. In addition to $\mathrm{CT}$, kinematic changes were observed in horizontal plane acceleration and included ML signal energy and AP impulse.

Significant changes identified in SF, VT impulse and AP signal energy at the end of the run, were most probably a result of an increased running velocity in the final stages of a run, rather than due to fatigue. 
Significant increases in CT were identified during the running protocol from laps four to 18, suggesting fatigued running throughout this time. Girard et al. [2] also observed significant increases in CT throughout a run (five $\mathrm{km}$ time trial) when compared to the original measurement. Other studies have also reported increases in CT with fatigue [4,12]. Significant increases in CT can be explained by a runner slowing their running velocity due to fatigue [2]. Although this study did not directly assess speed changes, research has identified when performing a time trial paced run it is a runner's natural tendency to progressively decrease running velocity throughout a major proportion of the run due to fatigue before significantly increasing velocity in the final stages [2]. When running fatigued at a slower running velocity, stride length and frequency decreases coinciding with the production of longer breaking and push off phases, increasing CT [2]. Increases in CT with fatigue has been suggested to be due to a deterioration in neuromuscular performance, resulting with compensatory longer push off durations due to reduced function of the stretch-shortening mechanism [14,15].

Girard et al. [2] reported smaller amplitudes of peak breaking and push off force when running under fatigue. As breaking and push off forces occur in the AP plane this may explain the significant decreases in AP impulse observed in this study, supporting these changes to represent a fatigued running biomechanical compensation. Whether reducing AP impulse when running fatigued reduces the risk of injury however is still yet to be determined. Significant changes in AP impulse were however identified to occur throughout prolonged run supporting the main finding of the study that biomechanical fatigue compensating running strategies are occurring throughout the duration of a run. Conflictingly, Le Bris et al. [3] did not identify any significant changes in AP impulse, and Schütte et al. [16] found AP accelerations increased with fatigue. However, the studies conducted by Le Bris et al. [3] and Schütte et al. [16] involved constant velocity running protocols, compared to self-selected pace run in this study, which may explain the differences in findings.

Fatigue also caused ML signal energy to significantly increase regularly throughout the run. Previous studies have also observed significant increases in ML acceleration kinematics with fatigue $[3,16]$. As running primarily occurs in the sagittal plane, the increase in the ML acceleration with fatigue were suggested to be due to a loss of coordination in this direction, which is not useful for propulsion [3], and may impact on running performance.

Step frequency, AP signal energy and VT impulse increased in the final stages of the run. This was most likely due to an increase in velocity rather than fatigue. While velocity measures were outside the scope of this study, Girard et al. [2] reported that runners increase their running velocity with subsequent increase in stride frequency in the final stages of a self-paced run [2].

\section{Implications and recommendations}

This study assessed data regularly throughout a prolonged run which informed us that not only fatigue related changes occurred, but how they occurred. Fatigue related running changes occurred in the early stages of a self-selected paced overground run and occurred continuously throughout. This suggests that runners are exposed to fatigue changes in the early stages of a run, but how this affects performance or is related to injury is unknown. This implies the need for strategies to minimize effects of fatigue from the onset of a run, which may include specific running technique or pacing strategies to minimize effects of fatigue. It is recommended that future research regularly assess data to enhance understanding about fatigue related changes and its impact on running performance and running related injuries.

\section{Limitations}

Limitations of this study include only investigating fatigue changes in male runners, therefore results may not be generalized to a female running population [17]. Another study limitation includes individual running skill level. This study included recreational through to competitive runners and literature has suggested that running kinematics significantly differ between different running skill levels, hence limiting the results of this study [8]. Although the results cannot be generalized to the entire distance running population, they still provide valuable information of running kinematic changes with fatigue.

\section{Conclusion}

A single tri-axial accelerometer identified that fatigue will impact a runner throughout a prolonged distance run. Horizontal plane accelerations AP impulse and ML signal energy were identified to significantly change in the early stages of the run and remained throughout, indicating alteration in running movement with fatigue. It is recommended that future research investigating the effects of fatigued running on running injuries and performance, perform frequent data collection and analysis to identify when and how fatigue running changes occur. A greater understanding of the impacts of fatigue may result in the development of fatigue minimizing preventative strategies to reduce the risk of running injuries and enhance running performance.

\section{Acknowledgements}

I would like to thank and acknowledge Alex Olsen for his assistance in creating the MATLAB code used for data analysis, and Daniel Lindsay for assisting in completing the statistical analysis. The authors of this research declare that the study compiled with the current laws of Australia and hold themselves responsible for all scientific context and legal aspects. There were no declared conflicts of interest in this study. 
Citation: Evans S, Winter S. Effects of fatigue on center-of-mass acceleration during a prolonged overground run. J Phys Ther Sports Med 2018;2(1):10-14.

\section{References}

1. Hreljac A. Impact and overuse injuries in runners. Med. Sci. Sports Exerc. 2004;36(5):845-9.

2. Girard O, Millet GP, Slawinski J, et al. Changes in running mechanics and spring-mass behaviour during a 5-km time trial. Int J Sports Med. 2013;34(09):832-40.

3. Le Bris R, Billat V, Auvinet B, et al. Effect of fatigue on stride pattern continuously measured by an accelerometric gait recorder in middle distance runners. J Sports Med Phys Fit. 2006;46(2):227.

4. Rabita G, Couturier A, Dorel S, et al. Changes in springmass behavior and muscle activity during an exhaustive run at V O2max. J Biomech. 2013;46(12):2011-7.

5. Mizrahi J, Verbitsky O, Isakov E, et al. Effect of fatigue on leg kinematics and impact acceleration in long distance running. Hum Mov Sci. 2000;19(2):139-51.

6. Dierks TA, Davis IS, Hamill J. The effects of running in an exerted state on lower extremity kinematics and joint timing. J Biomech. 2010;43(15):2993-8.

7. Hanley B, Mohan AK. Changes in gait during constant pace treadmill running. J Strength Cond Res. 2014;28(5): 1219-25.

8. Strohrmann C, Harms H, Kappeler-Setz C, et al. Monitoring kinematic changes with fatigue in running using body-worn sensors. IEEE Trans Inf Technol Biomed. 2012;16(5):983-90.

9. Bigelow EM, Elvin NG, Elvin AA, et al. Peak impact accelerations during track and treadmill running. J Appl Biomech. 2013;29(5):639-44.

10. McGregor SJ, Busa MA, Yaggie JA, et al. High resolution MEMS accelerometers to estimate $\mathrm{VO} 2$ and compare running mechanics between highly trained inter-collegiate and untrained runners. PLoS One. 2009;4(10):e7355.
11. Winter SC, Lee JB, Leadbetter RI, et al. Validation of a single inertial sensor for measuring running kinematics overground during a prolonged run. J Fit Res. 2016;5(1).

12. Rabita G, Slawinski J, Girard O, et al. Spring-mass behavior during exhaustive run at constant velocity in elite triathletes. Med Sci Sports Exerc. 2011;43(4):685-92.

13. Mathie MJ, Coster AC, Lovell NH, et al. Detection of daily physical activities using a triaxial accelerometer. Med Biol Eng Comput. 2003;41(3):296-301.

14. Komi PV. Stretch-shortening cycle: a powerful model to study normal and fatigued muscle. J Biomech. 2000;33(10): 1197-206.

15. Nicol C, Komi PV, Marconnet P. Fatigue effects of marathon running on neuromuscular performance. Scandinavian Journal of Medicine \& Science in Sports. 1991;1(1):10-7.

16. Schütte KH, Maas EA, Exadaktylos V, et al. Wireless triaxial trunk accelerometry detects deviations in dynamic center of mass motion due to running-induced fatigue. PloS one. 2015;10(10):e0141957.

17. Ferber R, Davis IM, Williams Iii DS. Gender differences in lower extremity mechanics during running. Clin Biomech. 2003;18(4):350-7.

\section{"Correspondence to:}

Scott Evans

James Cook University

E-mail:scott.evans@physioplus.com.au 